\title{
Kinetic lonic Permeation and Interfacial Doping of Supported Graphene
}

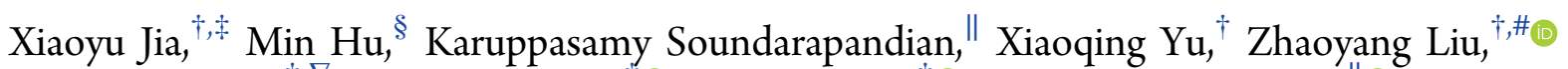
Zongping Chen, ${ }^{\dagger, \nabla}$ Akimitsu Narita, ${ }^{\dagger}$ Klaus Müllen, ${ }^{\dagger}$ Frank H. L. Koppens, ${ }^{\| \odot ~ J u n ~ J i a n g, ~}{ }^{\S \odot}$ Klaas-Jan Tielrooij, ${ }^{\perp}{ }^{+}$Mischa Bonn, ${ }^{\dagger}$ and Hai I. Wang* ${ }^{*} \dagger^{\dagger}(0)$

${ }^{\dagger}$ Max Planck Institute for Polymer Research, Ackermannweg 10, 55128 Mainz, Germany

${ }^{\ddagger}$ The Graduate School of Excellence Materials Science in Mainz, University of Mainz, Staudingerweg 9, 55128 Mainz, Germany

${ }^{\S}$ Hefei National Laboratory for Physical Sciences at the Microscale, iChEM (Collaborative Innovation Center of Chemistry for Energy Materials), CAS Center for Excellence in Nanoscience, School of Chemistry and Materials Science, University of Science and Technology of China, Hefei, Anhui 230026, China

"ICFO - Institut de Ciéncies Fotóniques, Mediterranean Technology Park, Castelldefels, Barcelona 08860, Spain

${ }^{\perp}$ Catalan Institute of Nanoscience and Nanotechnology (ICN2), BIST and CSIC, Campus UAB, Bellaterra, Barcelona 08193, Spain

Supporting Information

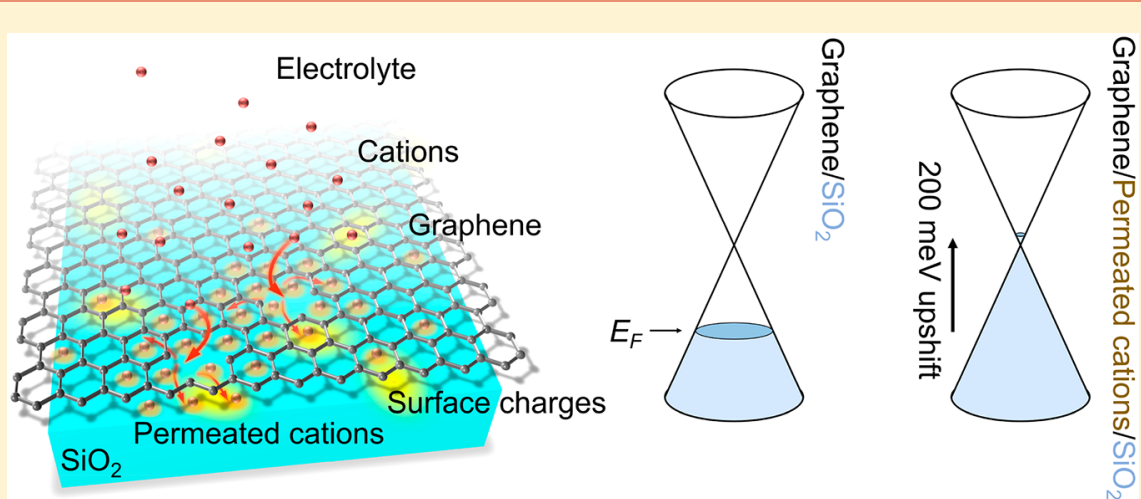

ABSTRACT: Due to its outstanding electrical properties and chemical stability, graphene finds widespread use in various electrochemical applications. Although the presence of electrolytes strongly affects its electrical conductivity, the underlying mechanism has remained elusive. Here, we employ terahertz spectroscopy as a contact-free means to investigate the impact of ubiquitous cations $\left(\mathrm{Li}^{+}, \mathrm{Na}^{+}, \mathrm{K}^{+}\right.$, and $\left.\mathrm{Ca}^{2+}\right)$ in aqueous solution on the electronic properties of $\mathrm{SiO}_{2}$-supported graphene. We find that, without applying any external potential, cations can shift the Fermi energy of initially hole-doped graphene by $\sim 200$ $\mathrm{meV}$ up to the Dirac point, thus counteracting the initial substrate-induced hole doping. Remarkably, the cation concentration and cation hydration complex size determine the kinetics and magnitude of this shift in the Fermi level. Combined with theoretical calculations, we show that the ion-induced Fermi level shift of graphene involves cationic permeation through graphene. The interfacial cations located between graphene and $\mathrm{SiO}_{2}$ electrostatically counteract the substrate-induced hole doping effect in graphene. These insights are crucial for graphene device processing and further developing graphene as an ionsensing material.

KEYWORDS: Graphene, terahertz spectroscopy, doping, ionic permeation

G raphene finds extensive use in various electrochemical applications, including ionic sensing, ${ }^{1,2}$ biochemical detection, ${ }^{3,4}$ lithium-ion batteries, ${ }^{5}$ dye-sensitized solar cells, ${ }^{6}$ and supercapacitors, ${ }^{7,8}$ due to its outstanding electrical and mechanical properties, large surface-to-volume ratio, and excellent chemical stability. Despite its critical importance for these applications, the effect of electrolyte solutions on the electronic properties of graphene has remained poorly understood. There is particular debate regarding the microscopic mechanism underlying the effect of aqueous solutions on graphene's conductivity, $\sigma=n e \mu$, where $n$ is the charge carrier density (related to the Fermi energy via $E_{\mathrm{F}}=\hbar \nu_{\mathrm{F}}(\pi|n|)^{1 / 2}$ where $\nu_{\mathrm{F}}$ is the Fermi velocity) and $\mu$ is the charge carrier mobility. Although it is apparent that the presence of (aqueous) electrolyte solutions affects the conductivity of graphene, it is typically not straightforward to disentangle how $\mu$ and $n$ are affected. In the case of polymer

Received: October 2, 2019

Revised: November 8, 2019

Published: November 19, 2019 
a)

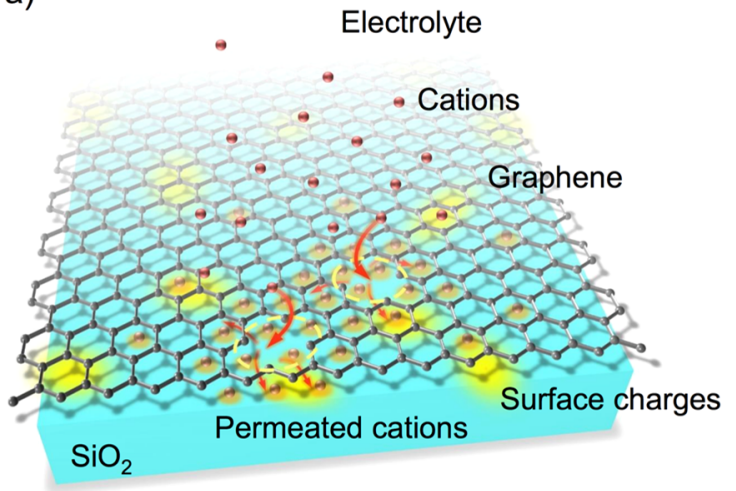

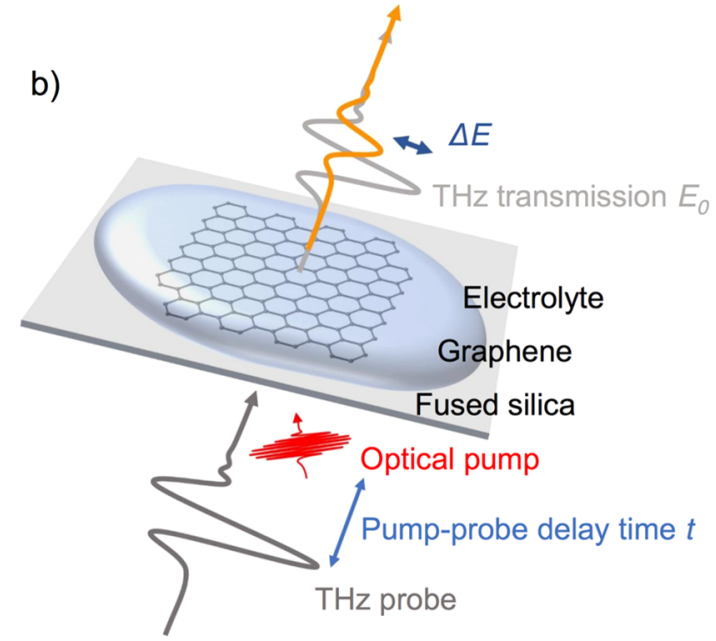

c)

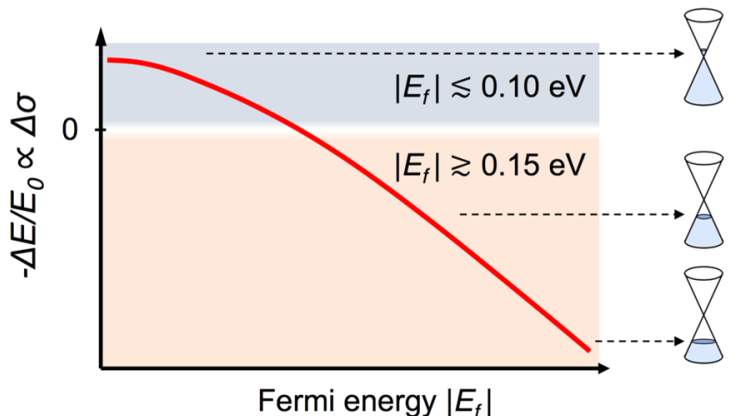

Figure 1. Concept of cation-induced Fermi level shift in graphene and its measurement. (a) Illustration of cation permeation through the graphene sheet to the graphene- $\mathrm{SiO}_{2}$ interface. Graphene is initially hole-doped due to the presence of negative surface charges (indicated in yellow) on the $\mathrm{SiO}_{2}$ surface. Intercalated cations lead to a shift of the Fermi level of graphene toward the Dirac point. (b) Schematic picture of optical pump-THz probe spectroscopy on the graphene/electrolyte system, with the graphene supported by fused silica $\left(\mathrm{SiO}_{2}\right)$. First, graphene is photoexcited by a femtosecond laser pulse (in red), followed by a $\mathrm{THz}$ probe pulse (in light gray). The change in $\mathrm{THz}$ transmission, $\Delta E=E-E_{0}$, is directly proportional to the change in $\mathrm{THz}$ conductivity, $\Delta \sigma$. (c) Illustration of typical Fermi energy-dependent $\mathrm{THz}$ photoconductivity, $-\Delta E / E_{0}$, in graphene. The conductivity is positive for graphene with the Fermi energy close to the Dirac point, whereas it becomes increasingly negative upon increasing the Fermi energy. ${ }^{25-28}$ This distinct sign change allows us to assign the effect of cations on the graphene conductivity to a change in Fermi level.

electrolyte solutions, it was found that they are extremely effective at inducing electron/hole doping in graphene through ions located in the Debye layer on top of graphene. ${ }^{9}$ The effect of cations has been primarily ascribed to cations absorbed on the surface of graphene, where they also act as chargescattering centers, ${ }^{10-12}$ thus affecting $\mu$. On the other hand, ions and water molecules have previously been reported to be able to diffuse between the interlayer space in van der Waals structures, ${ }^{13-16}$ and permeate through the graphene layer via atomic defects. ${ }^{17-19}$ Although these processes have been demonstrated to be useful for molecular separation and ionic sieving, ${ }^{17,20}$ its impact on the electronic and optoelectronic properties of graphene has not been considered and investigated. In addition, for graphene in contact with aqueous solutions, ion-induced variations of the charge carrier density, $n$, the role of defects in graphene, and the supporting substrate have remained elusive or mostly unexplored.

Here, employing optical pump-terahertz $(\mathrm{THz})$ probe (OPTP) spectroscopy as a noncontact, all-optical method to determine the conductivity, we investigate the impact of a series of cations (based on chloride salts $\mathrm{XCl}$ or $\mathrm{YCl}_{2}$, with $\mathrm{X}=$ $\mathrm{Li}^{+}, \mathrm{Na}^{+}, \mathrm{K}^{+}$, and $\mathrm{Y}=\mathrm{Ca}^{2+}$ ) in aqueous solution on the electrical conductivity of graphene supported by $\mathrm{SiO}_{2}$. We demonstrate that metal cations affect the conductivity mainly through a strong electrostatic effect that shifts the Fermi level toward the Dirac point $\left(\Delta E_{\mathrm{F}} \sim 200 \mathrm{meV}\right)$. Through kinetic measurements, we find that it takes tens of minutes for the Fermi energy shift to complete, where the time scale of this process depends critically on the solvation radius of the cations. We rationalize the concentration- and size-dependent cation-induced Fermi level shift that we observe as follows: cations permeate through pores or atomic defects in graphene (as schematically illustrated in Figure 1a) and diffuse further between the graphene- $\mathrm{SiO}_{2}$ interface. Through electrostatic effects, most likely involving screening of the negatively charged $\mathrm{SiO}_{2}$ surface by the intercalated cations, the p-type doping effect from the substrate is counteracted. Based on firstprinciples calculations, we find that, in line with our experimental observations, cations favor residing between graphene and $\mathrm{SiO}_{2}$ rather than on top of graphene. Our report reveals the process of cation permeability through graphene and its impact on the electronic properties of graphene, highlighting the importance of the interfacial chemistry for graphene's applications in electrochemistry and electronics. These results have implications for graphene-based ion-sensing devices, graphene device processing, and possible applications of graphene in desalination membranes. 


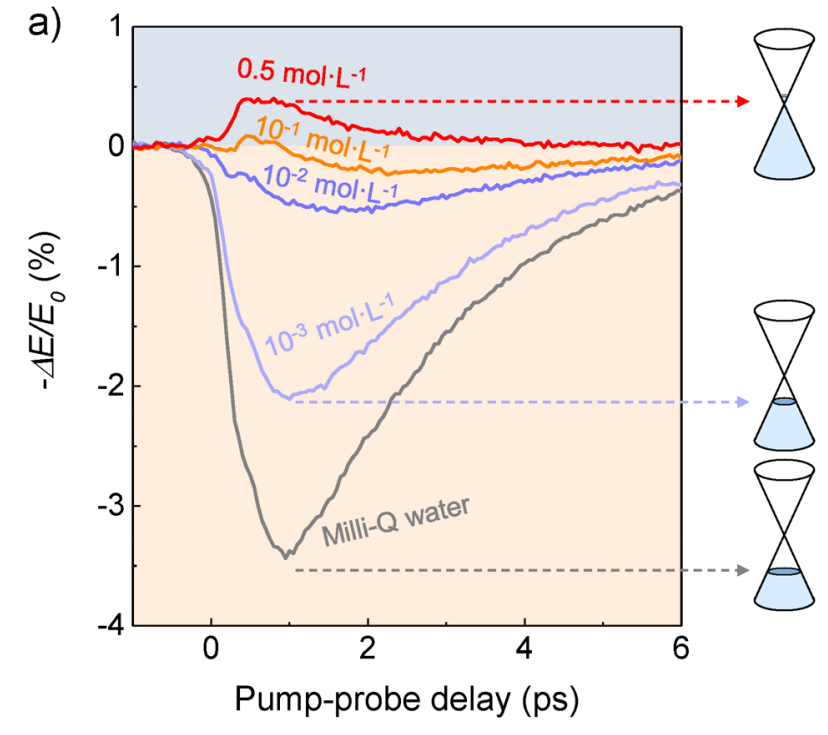

b)

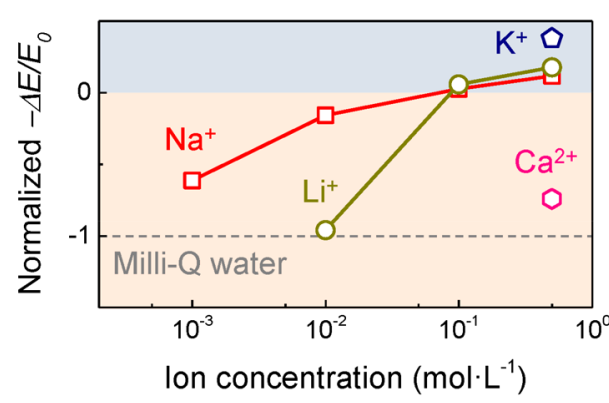

c)

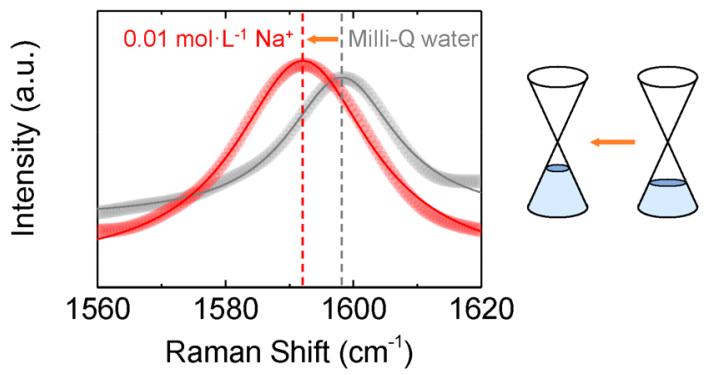

Figure 2. Effect of cations on the $\mathrm{THz}$ photoconductivity and Raman spectrum of graphene. (a) Cation $\left(\mathrm{Na}^{+}\right)$concentration-dependent $\mathrm{THz}$ conductivity $\left(-\Delta E / E_{0}\right)$ dynamics. The dynamics are recorded when the signal is saturated after graphene is in contact with electrolyte. (b) Peak value of the pump-induced $\mathrm{THz}$ conductivity for various ions $\left(\mathrm{Li}^{+}, \mathrm{Na}^{+}, \mathrm{K}^{+}, \mathrm{Ca}^{2+}\right)$ as a function of ion concentration. All data points are normalized to the THz conductivity value in Milli-Q water (shown as the gray dashed line). (c) Raman spectroscopy of graphene in the electrolyte. The G peak positions are $1592.1 \mathrm{~cm}^{-1}$ for graphene in $0.01 \mathrm{M} \mathrm{Na}^{+}$and $1598.2 \mathrm{~cm}^{-1}$ for Milli-Q. This indicates a shift of the Fermi level toward the Dirac point, as shown in the schematics on the right.

Results. We study the electrolyte effect on the electronic properties of graphene using monolayer graphene grown by chemical vapor deposition (CVD) that is transferred onto a fused silica $\left(\mathrm{SiO}_{2}\right)$ substrate (details in the Supporting Information). As typically observed, this leads to hole-doped graphene. The origin of this p-doping effect has been widely studied. Employing first-principles simulations, Nistor et al. identified the (negatively charged) dangling oxygen bonds present in the $\mathrm{SiO}_{2}$ substrate as one of the main sources. ${ }^{21}$ Other origins, including hole-donating impurities either in the substrate lattice or on the graphene surfaces (such as molecular $\mathrm{O}_{2}$ ), have been reported and proposed. ${ }^{22-24}$ We characterize the initial Fermi level of graphene on silica (without electrolyte) by Raman spectroscopy and find it to be $\left|E_{\mathrm{F}}\right|=$ 220-280 meV (below the Dirac point). We then bring the graphene in contact with electrolytes by sandwiching the electrolyte of interest between the graphene (supported by $\mathrm{SiO}_{2}$ ) and a top $\mathrm{SiO}_{2}$ glass; we limit the thickness of the electrolyte by a Teflon spacer to be $\sim 10 \mu \mathrm{m}$ to ensure sufficient $\mathrm{THz}$ transmission. In our study, the fluence of the laser pump is chosen such that we observe no change of $\mathrm{THz}$ conductivity of graphene over $1 \mathrm{~h}$ of measurements, as shown in Figure S1. This indicates that our technique is noninvasive and does not create new defects or increase the size of existing defects.

Size-Dependent Cation Induced Doping Effect in Graphene. To monitor how cations affect the graphene conductivity, we employ OPTP spectroscopy. In a typical OPTP measurement, an $800 \mathrm{~nm}$ laser pulse with sub-100 fs duration photoexcites graphene. Subsequently, we record the $\mathrm{THz}$ transmission change induced by this pump as a function of delay time $\mathrm{e}^{29}$ between the optical excitation and $\mathrm{THz}$ pulse (Figure 1b). The change in the transmitted THz field, $\Delta E=$
$E_{\text {pump }}-E_{0}$ (where $E_{\text {pump }}$ and $E_{0}$ are the transmissions with and without photoexcitation, respectively), is proportional to the conductivity change $(-\Delta E \propto \Delta \sigma)$ of graphene. Whereas this technique is a powerful tool to study the ultrafast charge dynamics and conductivity in graphene, ${ }^{25-28,30-34}$ it also allows for obtaining information on the steady-state Fermi energy in a rather straightforward manner. The reason for this is that the sign and magnitude of the pump-induced $\mathrm{THz}$ photoconductivity $\left(-\Delta E / E_{0}\right.$ or $\left.\Delta \sigma\right)$ are directly correlated to the Fermi energy in graphene, as we illustrate in Figure 1c: When the Fermi level is close to the Dirac point in graphene (I $E_{\mathrm{F}} \mathrm{I} \lesssim 0.10 \mathrm{eV}$ ), photoexcitation leads to graphene becoming more conductive; that is, the $\mathrm{THz}$ conductivity is positive following photoexcitation, whereas with the Fermi level away from the Dirac point $\left(\left|E_{\mathrm{F}}\right| \gtrsim 0.15 \mathrm{eV}\right)$, graphene becomes more resistive after the optical pump, so the $\mathrm{THz}$ conductivity is negative. $^{25-28,30,32,33}$ Thus, by monitoring the sign and magnitude of the changes in $\mathrm{THz}$ conductivity, we readily obtain the modulation of the Fermi energy in graphene. Importantly, this enables us to unambiguously disentangle changes in carrier density from changes in carrier mobility.

To interrogate the effect of the electrolyte or, more specifically, the role of hydrated cations in aqueous solution on the carrier conductivity in graphene, we study four different metal chloride solutions with various cation sizes: $\mathrm{KCl}, \mathrm{NaCl}$, $\mathrm{LiCl}$, and $\mathrm{CaCl}_{2}$. To quantitatively study the effect of cations on the Fermi level, we fix the anion to $\mathrm{Cl}^{-}$and the solvent to $\mathrm{H}_{2} \mathrm{O}$, thus focusing on the effect of cations. First, we investigate the effect of ionic concentration on the pumpinduced $\mathrm{THz}$ conductivity (referred to as " $\mathrm{THz}$ photoconductivity" below) of graphene for the four different electrolytes. The electrolyte solutions do not absorb the excitation light and therefore show no differential terahertz 

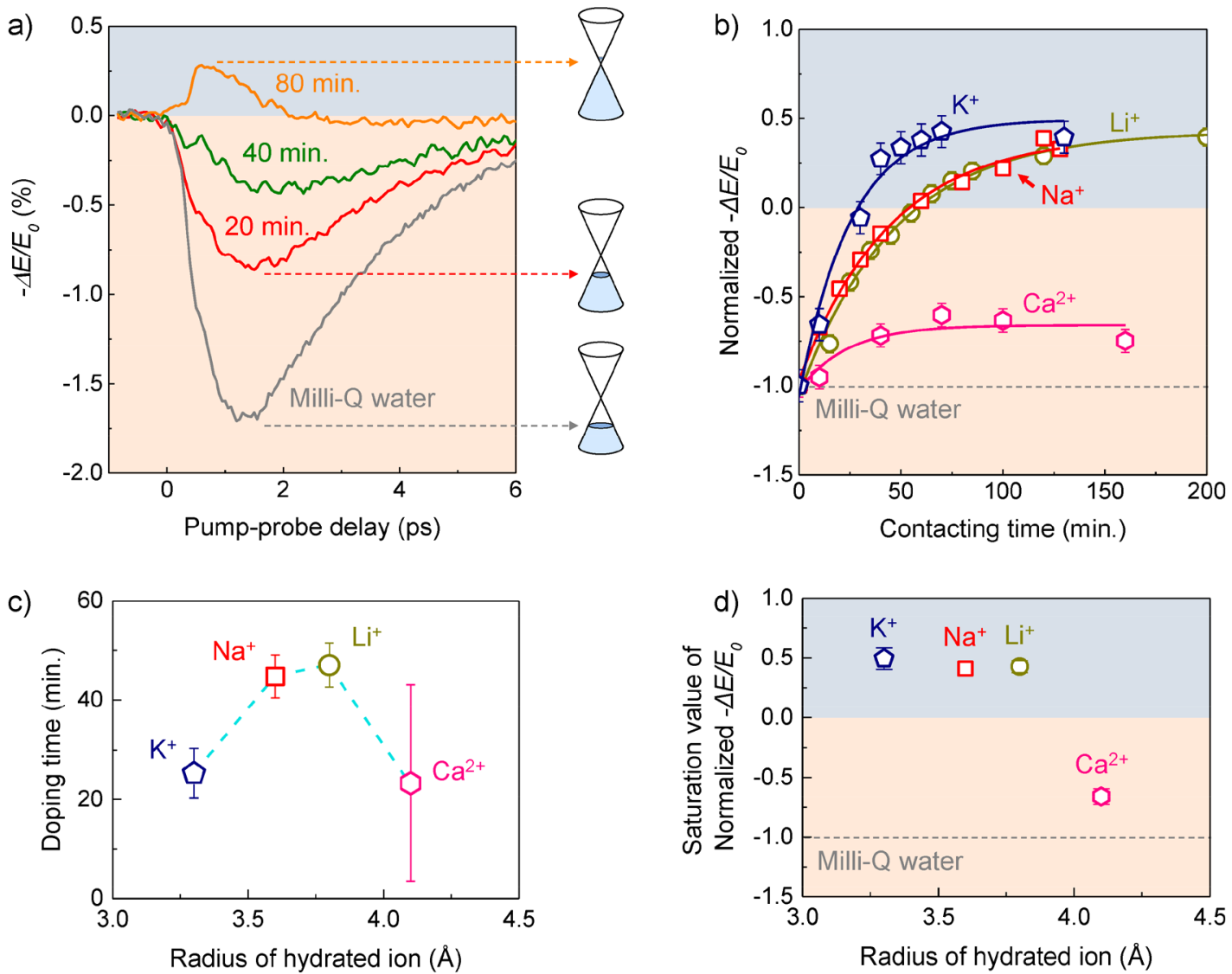

Figure 3. Time evolution of the cation-induced Fermi level shift in graphene. (a) Kinetic sodium ion $\left(\mathrm{Na}^{+}\right)$-induced Fermi level shift in graphene, as revealed by monitoring the pump-induced change in $\mathrm{THz}$ transmission depending on the contacting time of sodium ion solution, $0.5 \mathrm{M} \mathrm{NaCl}$ aqueous solution. All measurements are conducted in the same spot of a graphene sample. (b) Peak value of the pump-induced THz change, $-\Delta E$ / $E_{0}$, as a function of contacting time for metal cations including $\mathrm{K}^{+}, \mathrm{Na}^{+}, \mathrm{Li}^{+}$, and $\mathrm{Ca}^{2+}$. All data points are normalized to the same starting point for a better comparison. (c) Doping time is plotted as the function of the radius of the hydrated ion. (d) Archived final Fermi levels (representing by the cation-induced $\mathrm{THz}$ conductivity changes in graphene) vs the hydrated cation radius.

response. We show typical results for $\mathrm{NaCl}$ in Figure 2a: the electrolyte gives rise to a decrease in the magnitude of the negative conductivity in graphene, using the $\mathrm{THz}$ photoconductivity of graphene in contact with Milli-Q water as a reference. At sufficiently high concentrations, the sign of the photoconductivity becomes positive $(>0.1 \mathrm{M}$ for $\mathrm{NaCl}$, as presented in Figure 2a). Following the discussion related to Figure $1 \mathrm{~b}$, and given the fact that the initial doping of our graphene is p-type $(\sim 220-280 \mathrm{meV}$ below the Dirac point), we conclude that the $\mathrm{NaCl}$ electrolyte induces a shift of the Fermi level toward the Dirac point (i.e., less hole doping) by an amount $\Delta E_{\mathrm{F}} \sim 200 \mathrm{meV}$.

As an independent verification of the observations using OPTP measurements, we perform Raman measurements, which allow for extracting the change of Fermi level in graphene. As shown in Figure 2c, we observe a shift of the G band from $1598.2 \mathrm{~cm}^{-1}$ for graphene in contact with $0.01 \mathrm{M}$ $\mathrm{NaCl}$ electrolyte to $1592.1 \mathrm{~cm}^{-1}$ for graphene in contact with Milli-Q water, corresponding to a shift of Fermi level of $\sim 150$ $\mathrm{meV}$ toward the Dirac point, in agreement with our OPTP results ( $200 \mathrm{meV}$ upshift of the Fermi level in graphene by $0.5 \mathrm{M} \mathrm{NaCl}$ electrolyte).

In the following, we vary the cation concentration and study how this affects the $\mathrm{THz}$ photoconductivity with respect to that of graphene in contact with Milli- $Q$ water as a reference. We summarize the cation-induced effect on the $\mathrm{THz}$ photoconductivity of graphene (by taking the peak values charge carrier dynamics shown in Figure $2 a$ ) for a series of cations with varying ionic concentration, as shown in Figure 2b. Whereas all electrolytes show qualitatively the same effect (less negative photoconductivity and thus a Fermi energy shift toward the Dirac point), the achieved final Fermi level in graphene displays an obvious cation size effect with a trend from the largest shift for $\mathrm{K}^{+}$(with the smallest solvation radius of $\sim 3.3 \AA$ among all cations studied here ${ }^{35}$ ) to the smallest change for $\mathrm{Ca}^{2+}$ (with the largest solvation radius of $\sim 4.1 \AA$ for the cations used ${ }^{35}$ ). Sodium and lithium ions with similar size of hydrated radius have comparable effects at high concentration. In our study, we use the hydrated ion (solvation) radius (defined as the radius of the hydration sphere of the ions) rather than the ion radius because the solvation radius is relevant for transport in an aqueous solution.

Tracking the Cation Induced Doping Kinetics in Graphene. To further investigate the underlying mechanism for the observed size-dependent cation effect on the Fermi level, we monitor in situ the kinetics of the Fermi level changes by tracking the $\mathrm{THz}$ photoconductivity as a function of graphene-electrolyte contacting time. In Figure 3a for graphene in contact with a $0.5 \mathrm{M}$ sodium chloride solution, the ion-induced Fermi level shift is not an instantaneous process. Instead, it takes $\sim 80 \mathrm{~min}$ for the $\mathrm{THz}$ conductivity of graphene to gradually turn from negative to positive 


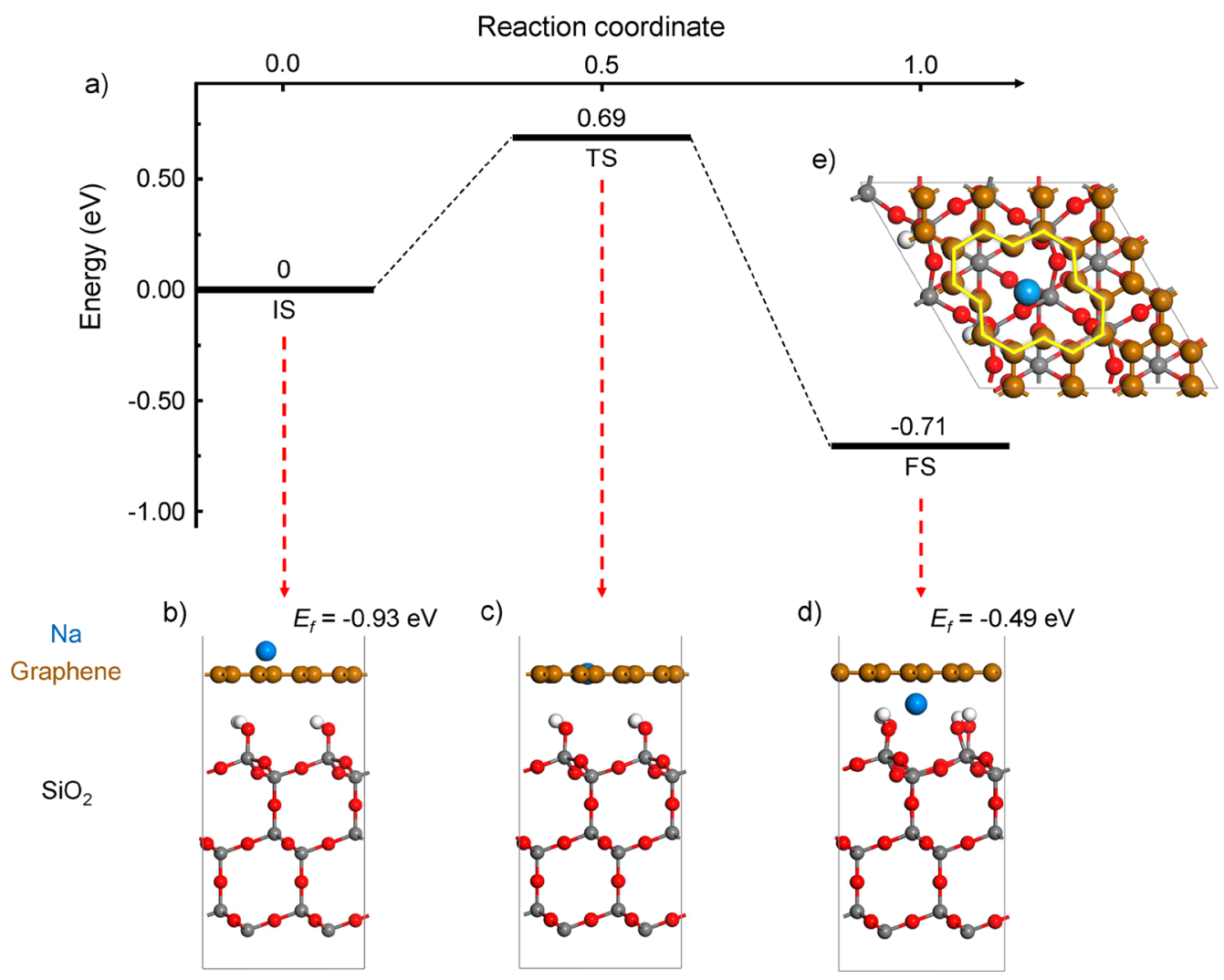

Figure 4. Simulation of cation effect at the graphene/ $\mathrm{SiO}_{2}$ interface. (a) System energetics for $\mathrm{Na}$ adsorbing at graphene's top surface, the energy barrier for Na permeation through a graphene double vacancy defect, and $\mathrm{Na}$ residing at the graphene- $\mathrm{SiO}_{2}$ substrate interfaces. (b-d) Modeled atomic structures in cross-sectional views corresponding to the three energy states in (a). When $\mathrm{Na}_{\text {is }}$ at graphene's top surface and at $\mathrm{SiO}_{2}$ 's surface beneath graphene, the calculated Fermi energies of the system are -0.93 and $-0.49 \mathrm{eV}$, respectively (details in Figure S6). (e) Na residing at graphene- $\mathrm{SiO}_{2}$ substrate interfaces after it permeates through a double vacancy defect (highlighted as a yellow polygon).

(corresponding to a change from hole-doped graphene to graphene with a Fermi energy in the vicinity of the Dirac point). We also study these cation-induced dynamics for graphene in contact with cations of different concentrations and sizes. First, we measure the ionic effect induced by $10^{-2}$ and $0.5 \mathrm{M} \mathrm{NaCl}$ electrolyte at the same sample spot of a graphene sample, as demonstrated in Figure S2a. The time required for reaching the final Fermi energy is much shorter for increased concentrations ( $\sim 80 \mathrm{~min}$ vs $150 \mathrm{~min}$ to change the $\mathrm{THz}$ conductivity sign from negative to positive for $0.5 \mathrm{M}$ in comparison to $10^{-2} \mathrm{M} \mathrm{NaCl}$, respectively). Second, we compare concentration-dependent $\mathrm{THz}$ conductivity measurements for $\mathrm{K}^{+}, \mathrm{Li}^{+}$, and $\mathrm{Ca}^{2+}$. For a reliable comparison, we conduct all the $\mathrm{THz}$ measurements in a single spot of a graphene sample and fix the cationic concentration to be $0.5 \mathrm{M}$ for all cases (for all original data, see Figure S2b in the Supporting Information). We describe the normalized timedependent dynamics of the photoconductivity change by a single-exponential process, as indicated by the solid lines in Figure $3 \mathrm{~b}$. We plot the inferred exponential time scales and the final photoconductivity as a function of the solvation radius of the cations in Figure 3c,d. It appears that all three small cations $\left(\mathrm{Li}^{+}, \mathrm{Na}^{+}, \mathrm{K}^{+}\right)$used here can effectively modify the carrier density of graphene. However, cations with a larger ionic solvation radius $\left(\mathrm{K}^{+}<\mathrm{Na}^{+}<\mathrm{Li}^{+}\right)$require an increasingly longer time to achieve the Fermi level shift. For the largest hydrated ions, $\mathrm{Ca}^{2+}$, we observe a rather slight change in graphene's conductivity and, thus, Fermi energy. Finally, it is worth commenting that although the trend of the size- dependent kinetics and degree of Fermi energy shift induced by cations (as shown in Figure 3c,d) is highly reproducible for all samples, we find that the absolute changes (e.g., doping time and magnitude) vary from sample to sample, presumably due to the variations in initial doping and defect densities of graphene in different samples (see the extended discussion in the next section).

Ionic Permeation and Interfacial Doping of Supported Graphene. The observed cation-induced change in Fermi energy (more specifically, the reduced hole density) challenges the intuitive and straightforward view that the cations are located on the top surface of graphene (which would not give rise to the observed slow, size- and time-dependent doping processes), and that they act only passively as scattering centers, solely modifying the carrier mobility. Instead, our results here can be explained by an ionic process that involves concentration-, size-, and time-dependent permeation of metal cations through atomic defects in graphene. ${ }^{17-19}$ The intercalated cations between the graphene and substrate surfaces then electrostatically screen the p-doping effect of the $\mathrm{SiO}_{2}$ substrate, thus resulting in graphene with reduced $\mathrm{p}$ doping. In principle, an atomically perfect graphene lattice is impermeable for all ions except for protons $\left(\mathrm{H}^{+}\right)$, thus acting as an ideal sieve to protons from other cations. ${ }^{20}$ This picture, however, does not hold for large-area graphene with inevitable atomic defects, such as missing carbon atoms resulting in nanopores. Indeed, permeation of ions and water molecules through graphene atomic defects has previously been reported, ${ }^{19-19}$ and selective cation transport through graphene 
nanopores has also been demonstrated. ${ }^{17-19,36-38}$ Moreover, based on atomic force microscopy measurements of the CVD graphene used here (see Figure S3), we find grain boundaries and holes in the size range of up to several micrometers, which can be the initial sites for cation intercalation. As such, cation permeation is kinetically possible in our CVD graphene and is thermodynamically favorable (which is further supported by the simulation discussion in Figure 4). The cations tend to move to the supported silica surfaces and remain intercalated between graphene and the silica substrate, due to the negative surface potential of silica at neutral $\mathrm{pH}$. The permeated cations can subsequently diffuse in-plane, away from the defect centers, along the graphene- $\mathrm{SiO}_{2}$ interface.

For a certain size and amount of atomic defects in graphene, ionic permeation, as well as the subsequent diffusion of ions intercalated between graphene and $\mathrm{SiO}_{2}$, depends critically on the size of the hydrated ions. ${ }^{18,19,36-38}$ Therefore, we expect cationic permeation and in-plane diffusion to speed up with smaller solvated ion size, as we indeed observe. When the size of hydrated cations or a hydrated cation complex (as for the case of $\mathrm{Ca}^{2+39}$ ) further increases to be comparable or larger than the defects or nanopore size in graphene, ionic blockade can take place, ${ }^{36}$ also in accordance with our experiment.

To further support our explanation and model of ionic permeation and the resultant shift of the Fermi energy of graphene by the intercalated cations, we highlight two additional experimental observations. First, following the equilibration of the graphene/silica system with a specific electrolyte solution, we exchange that solution with pure water and investigate the reverse process of the Fermi level shift ("redoping") (Figure S2c). We find that substrate-induced hole doping is recovered after rinsing away the solutions containing the cations $\mathrm{Li}^{+}, \mathrm{Na}^{+}$, and $\mathrm{K}^{+}$. The recovery of the doping level in the absence of ions exhibits distinct sizedependent kinetics (detailed discussion in Figure S2). If the cations would not intercalate between $\mathrm{SiO}_{2}$ and graphene, we would expect the ions to dissolve very quickly back into the water when the graphene is contacted with pure water. Instead, we observe a time scale on the order of tens of minutes and a clear dependence on ion hydration size, thus corroborating our picture of intercalated cations. Second, we find that the relative change in $\mathrm{THz}$ conductivity (and thus Fermi level shift) for a given ionic composition and concentration is much more pronounced when the graphene contains fewer polymer residues (by comparing two sets of graphene samples using different post-transfer treatments; details in Figure S4). We rationalize that the Fermi level shift depends crucially on the sample cleanness; that is, when the cation permeation pathway is blocked by the polymer residuals, a smaller change in carrier density in graphene is achieved.

Simulations. We now present first-principles calculations by which we explore the interaction between $\mathrm{SiO}_{2}$-supported graphene and electrolyte solutions. The simulations are based on spin-polarized density functional theory calculations as implemented in the Vienna ab initio simulation package, ${ }^{40,41}$ using Perdew-Burke-Ernzerhof ${ }^{42}$ functionals and the planewave projector augmented wave ${ }^{43}$ method with van der Waals interactions.

In the optimized structure of supported graphene, the $\mathrm{Si}-\mathrm{O}$ bond lengths are in the range of 1.60-1.62 $\AA$, in good agreement with those in previous studies. ${ }^{44,45}$ The binding energy of $-0.84 \mathrm{eV}$ for a unit cell of the complex system is calculated as the energy difference between the bonded system and the isolated parts, suggesting high stability. We then add a $\mathrm{Na}$ atom (consisting of $\mathrm{Na}^{+}$ion plus an electron) into the model system to investigate the cation effect on graphene. To simplify the calculation, here, only $\mathrm{Na}^{+}$is included in the simulation, and charge neutralization is realized by adding an electron instead of a $\mathrm{Cl}^{-}$anion. Despite the simplicity of our model, it captures the essence of the cation-graphene interaction and explains our experimental data well (as shown below). First, we investigate the energetics for two configurations of $\mathrm{Na}^{+}$either on top of graphene or residing between graphene and the $\mathrm{SiO}_{2}$ support. As shown in Figure $4 a$, our results clearly illustrate that it is energetically more favorable for the cation to reside between graphene and $\mathrm{SiO}_{2}$ than to be adsorbed at the top surface of graphene, in good agreement with the conclusions from the experiments. Furthermore, we explore the kinetic $\mathrm{Na}^{+}$permeation processes for graphene with (e.g., single vacancies and double vacancies) and without atomic defects (detailed in the Supporting Information). The permeation of $\mathrm{Na}^{+}$through a perfect graphene layer is associated with a prohibitively high energetic barrier $(>10 \mathrm{eV})$. The presence of a double vacancy reduces the barrier to $0.69 \mathrm{eV}$ (3.22 eV with a single vacancy, as shown in Figure S5), which is comparable to the penetration barrier of $1.2-1.6 \mathrm{eV}$ for a proton to migrate through the perfect graphene monolayer. ${ }^{20}$ Finally, to investigate the impact of intercalating cations on graphene, we compute the density of states (DOS) of the complex with and without $\mathrm{Na}^{+}$(Figure S6). The Fermi level appreciably shifts toward less negative values (from -0.93 to $-0.49 \mathrm{eV}$ ) when $\mathrm{Na}^{+}$is included in the complex. Figure S7 shows the partial DOS of the C atoms around $\mathrm{Na}^{+}$. Substantial p-orbitals of these carbon atoms emerge in the vicinity of the up-shifted Fermi level, suggesting that the new filled state originates from electrons in the porbitals of the $\mathrm{C}$ atoms in the vicinity of interfacial $\mathrm{Na}^{+}$. The shift of Fermi level in the complex system induced by $\mathrm{Na}^{+}$is in line with the experimental results.

Summary. To summarize, employing optical pump-THz probe spectroscopy as a contact-free, all-optical conductivity measurement, we investigate the role of a series of cations with various solvation radii (based on chloride salt $\mathrm{XCl}$ and $\mathrm{YCl}_{2}$ with $\mathrm{X}=\mathrm{Li}^{+}, \mathrm{Na}^{+}, \mathrm{K}^{+}$, and $\mathrm{Y}=\mathrm{Ca}^{2+}$ ) on the electrical conductivity of graphene. We observe a cation-induced shift of the Fermi level in excess of $200 \mathrm{meV}$ toward the Dirac point in graphene. Combined with theoretical calculations, such an ionic effect in graphene appears to involve cationic permeation through defects in graphene, which is kinetically controlled by the interplay of the hydration radius of cations and the size of atomic defects in graphene.

These findings not only provide a new tool to monitor the ionic dynamics at graphene and potentially other materials interfaces but also offer new insights into the role of cation permeability through graphene and the influence of ion interfacial dynamics on electronic properties. Finally, these results are of interest for electrochemical charge and energy storage, graphene device processing (where, for example, etching chemical or electrolytes are extensively in use), desalination membranes, and ionic-sensing devices. Regarding the last case, controlling the size and density of atomic defects in graphene, as well as the nature of surface charges in supporting substrates, can serve as effective and novel approaches to enhance the ionic sensitivity and specificity. 


\section{ASSOCIATED CONTENT}

\section{S Supporting Information}

The Supporting Information is available free of charge at https://pubs.acs.org/doi/10.1021/acs.nanolett.9b04053.

Optical pump- $\mathrm{THz}$ probe spectroscopy; graphene transfer; electrolyte contacting; computational methods and details; stability of graphene in laser excitation; evolution of the cation doping dynamics on graphene; surface characterization of graphene on $\mathrm{SiO}_{2}$; comparison between less-clean graphene and clean graphene on fused silica; energy landscape and modeled structures for $\mathrm{Na}^{+}$ion penetrating vacancies; density of state calculation result of graphene- $\mathrm{SiO}_{2}$ interface; calculated PDOS of the $\mathrm{C}$ atom around $\mathrm{Na}^{+}$(PDF)

\section{AUTHOR INFORMATION}

\section{Corresponding Author}

*E-mail: wanghai@mpip-mainz.mpg.de.

\section{ORCID $\odot$}

Zhaoyang Liu: 0000-0001-7972-3452

Akimitsu Narita: 0000-0002-3625-522X

Klaus Müllen: 0000-0001-6630-8786

Frank H. L. Koppens: 0000-0001-9764-6120

Jun Jiang: 0000-0002-6116-5605

Klaas-Jan Tielrooij: 0000-0002-0055-6231

Mischa Bonn: 0000-0001-6851-8453

Hai I. Wang: 0000-0003-0940-3984

\section{Present Addresses}

${ }^{\#}$ Université de Strasbourg, CNRS, ISIS, 8 Alleé Gaspard Monge, 67000 Strasbourg, France.

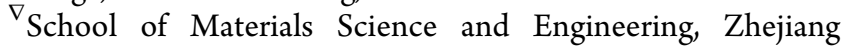
University, 38 Zheda Road Hangzhou 310027, China.

\section{Author Contributions}

H.I.W. and X.J. conceived the research and wrote the paper with input from all authors. H.I.W., K.-J.T., and M.B. supervised the project and interpreted the data. K.S., X.J., and F.H.L.K. prepared and characterized the graphene samples. Z.L., Z.C., A.N., and K.M. helped with the graphene sample preparation. X.J. obtained and analyzed the Raman and OPTP spectroscopy data. X.Y. helped with OPTP spectroscopy measurements. M.H. and J.J. performed the first-principles simulations.

\section{Notes}

The authors declare no competing financial interest.

\section{ACKNOWLEDGMENTS}

We thank Euan Hendry, Yuki Nagata, Ellen Backus, Lisa Dreier, Mathias Kläui, and Jose A. Garrido for fruitful discussions. We also thank Eduard Unger and Marco Ballabio for help with THz measurements. X.J., Z.L., Z.C., A.N., K.M., M.B., and H.I.W. acknowledge the financial support by the Max Planck Society. X.J. acknowledges support by a Deutsche Forschungsgemeinschaft-funded position through the Excellence Initiative by the Graduate School of Excellence Materials Science in Mainz (MAINZ) (GSC 266) and support from the Max Planck Graduate Center mit der Johannes GutenbergUniversität Mainz (MPGC). K.-J.T. acknowledges financial support through the MAINZ Visiting Professorship. X.Y. acknowledges the fellowship support from Chinese Scholarship Council (CSC). This project has received funding from the
European Union's Horizon 2020 research and innovation program under Grant Agreement No. 804349. ICN2 is supported by the Severo Ochoa program from Spanish MINECO (Grant No. SEV-2017-0706). F.H.L.K. acknowledges financial support by Fundacio Cellex Barcelona, support from the Spanish Ministry of Economy and Competitiveness, through the "Severo Ochoa" Programme for Centres of Excellence in R\&D (SEV-2015-0522), and support by the Generalitat de Catalunya through the CERCA program.

\section{REFERENCES}

(1) Fu, W.; Jiang, L.; van Geest, E. P.; Lima, L. M. C.; Schneider, G. F. Sensing at the Surface of Graphene Field-Effect Transistors. Adv. Mater. 2017, 29 (6), 1603610.

(2) Pfattner, R.; Foudeh, A. M.; Chen, S.; Niu, W.; Matthews, J. R.; He, M.; Bao, Z. Dual-Gate Organic Field-Effect Transistor for PH Sensors with Tunable Sensitivity. Adv. Electron. Mater. 2019, 5 (1), 1800381.

(3) Shao, Y.; Wang, J.; Wu, H.; Liu, J.; Aksay, I. A.; Lin, Y. Graphene Based Electrochemical Sensors and Biosensors: A Review. Electroanalysis 2010, 22 (10), 1027-1036.

(4) Liu, Y.; Dong, X.; Chen, P. Biological and Chemical Sensors Based on Graphene Materials. Chem. Soc. Rev. 2012, 41 (6), 22832307.

(5) Kucinskis, G.; Bajars, G.; Kleperis, J. Graphene in Lithium Ion Battery Cathode Materials: A Review. J. Power Sources 2013, 240, 6679.

(6) Wang, X.; Zhi, L.; Müllen, K. Transparent, Conductive Graphene Electrodes for Dye-Sensitized Solar Cells. Nano Lett. 2008, 8 (1), 323-327.

(7) Weng, Z.; Su, Y.; Wang, D.-W.; Li, F.; Du, J.; Cheng, H.-M. Graphene-Cellulose Paper Flexible Supercapacitors. Adv. Energy Mater. 2011, 1 (5), 917-922.

(8) Liu, Z.; Wang, H. I.; Narita, A.; Chen, Q.; Mics, Z.; Turchinovich, D.; Kläui, M.; Bonn, M.; Müllen, K. Photoswitchable Micro-Supercapacitor Based on a Diarylethene-Graphene Composite Film. J. Am. Chem. Soc. 2017, 139 (28), 9443-9446.

(9) Das, A.; Pisana, S.; Chakraborty, B.; Piscanec, S.; Saha, S. K.; Waghmare, U. V.; Novoselov, K. S.; Krishnamurthy, H. R.; Geim, A. K.; Ferrari, A. C.; et al. Monitoring Dopants by Raman Scattering in an Electrochemically Top-Gated Graphene Transistor. Nat. Nanotechnol. 2008, 3 (4), 210-215.

(10) Newaz, A. K. M.; Puzyrev, Y. S.; Wang, B.; Pantelides, S. T.; Bolotin, K. I. Probing Charge Scattering Mechanisms in Suspended Graphene by Varying Its Dielectric Environment. Nat. Commun. 2012, 3, 734 .

(11) Sharma, P.; Mišković, Z. L. Ionic Screening of Charged Impurities in Electrolytically Gated Graphene: A Partially Linearized Poisson-Boltzmann Model. J. Chem. Phys. 2015, 143 (13), 134118.

(12) Hu, G.; Pandey, G. P.; Liu, Q.; Anaredy, R. S.; Ma, C.; Liu, M.; Li, J.; Shaw, S. K.; Wu, J. Self-Organization of Ions at the Interface between Graphene and Ionic Liquid DEME-TFSI. ACS Appl. Mater. Interfaces 2017, 9 (40), 35437-35443.

(13) Yao, F.; Güneş, F.; Ta, H. Q.; Lee, S. M.; Chae, S. J.; Sheem, K. Y.; Cojocaru, C. S.; Xie, S. S.; Lee, Y. H. Diffusion Mechanism of Lithium Ion through Basal Plane of Layered Graphene. J. Am. Chem. Soc. 2012, 134 (20), 8646-8654.

(14) Kühne, M.; Paolucci, F.; Popovic, J.; Ostrovsky, P. M.; Maier, J.; Smet, J. H. Ultrafast Lithium Diffusion in Bilayer Graphene. Nat. Nanotechnol. 2017, 12 (9), 895-900.

(15) Ji, K.; Han, J.; Hirata, A.; Fujita, T.; Shen, Y.; Ning, S.; Liu, P.; Kashani, H.; Tian, Y.; Ito, Y.; et al. Lithium Intercalation into Bilayer Graphene. Nat. Commun. 2019, 10 (1), 275.

(16) Kühne, M.; Börrnert, F.; Fecher, S.; Ghorbani-Asl, M.; Biskupek, J.; Samuelis, D.; Krasheninnikov, A. V.; Kaiser, U.; Smet, J. H. Reversible Superdense Ordering of Lithium between Two Graphene Sheets. Nature 2018, 564 (7735), 234-239. 
(17) O’Hern, S. C.; Boutilier, M. S. H.; Idrobo, J.-C.; Song, Y.; Kong, J.; Laoui, T.; Atieh, M.; Karnik, R. Selective Ionic Transport through Tunable Subnanometer Pores in Single-Layer Graphene Membranes. Nano Lett. 2014, 14 (3), 1234-1241.

(18) Achtyl, J. L.; Unocic, R. R.; Xu, L.; Cai, Y.; Raju, M.; Zhang, W.; Sacci, R. L.; Vlassiouk, I. V.; Fulvio, P. F.; Ganesh, P.; et al. Aqueous Proton Transfer across Single-Layer Graphene. Nat. Commun. 2015, 6, 6539.

(19) Esfandiar, A.; Radha, B.; Wang, F. C.; Yang, Q.; Hu, S.; Garaj, S.; Nair, R. R.; Geim, A. K.; Gopinadhan, K. Size Effect in Ion Transport through Angstrom-Scale Slits. Science 2017, 358 (6362), 511-513.

(20) Hu, S.; Lozada-Hidalgo, M.; Wang, F. C.; Mishchenko, A.; Schedin, F.; Nair, R. R.; Hill, E. W.; Boukhvalov, D. W.; Katsnelson, M. I.; Dryfe, R. a. W.; et al. Proton Transport through One-AtomThick Crystals. Nature 2014, 516 (7530), 227-230.

(21) Nistor, R. A.; Kuroda, M. A.; Maarouf, A. A.; Martyna, G. J. Doping of Adsorbed Graphene from Defects and Impurities in $\mathrm{SiO}_{2}$ Substrates. Phys. Rev. B: Condens. Matter Mater. Phys. 2012, 86 (4), 041409.

(22) Ryu, S.; Liu, L.; Berciaud, S.; Yu, Y.-J.; Liu, H.; Kim, P.; Flynn, G. W.; Brus, L. E. Atmospheric Oxygen Binding and Hole Doping in Deformed Graphene on $\mathrm{SiO}_{2}$ Substrate. Nano Lett. 2010, 10 (12), 4944-4951.

(23) Hornett, S. M.; Heath, M.; Horsell, D. W.; Hendry, E. Optically Induced Oxygen Desorption from Graphene Measured Using Femtosecond Two-Pulse Correlation. Phys. Rev. B: Condens. Matter Mater. Phys. 2014, 90 (8), 081401.

(24) Piazza, A.; Giannazzo, F.; Buscarino, G.; Fisichella, G.; Magna, A. L.; Roccaforte, F.; Cannas, M.; Gelardi, F. M.; Agnello, S. Graphene p-Type Doping and Stability by Thermal Treatments in Molecular Oxygen Controlled Atmosphere. J. Phys. Chem. C 2015, 119 (39), 22718-22723.

(25) Shi, S.-F.; Tang, T.-T.; Zeng, B.; Ju, L.; Zhou, Q.; Zettl, A.; Wang, F. Controlling Graphene Ultrafast Hot Carrier Response from Metal-like to Semiconductor-like by Electrostatic Gating. Nano Lett. 2014, 14 (3), 1578-1582.

(26) Frenzel, A. J.; Lui, C. H.; Shin, Y. C.; Kong, J.; Gedik, N. Semiconducting-to-Metallic Photoconductivity Crossover and Temperature-Dependent Drude Weight in Graphene. Phys. Rev. Lett. 2014, 113 (5), 056602.

(27) Jensen, S. A.; Mics, Z.; Ivanov, I.; Varol, H. S.; Turchinovich, D.; Koppens, F. H. L.; Bonn, M.; Tielrooij, K. J. Competing Ultrafast Energy Relaxation Pathways in Photoexcited Graphene. Nano Lett. 2014, 14 (10), 5839-5845.

(28) Tomadin, A.; Hornett, S. M.; Wang, H. I.; Alexeev, E. M.; Candini, A.; Coletti, C.; Turchinovich, D.; Kläui, M.; Bonn, M.; Koppens, F. H. L.; et al. The Ultrafast Dynamics and Conductivity of Photoexcited Graphene at Different Fermi Energies. Sci. Adv. 2018, 4 (5), No. eaar5313.

(29) Ulbricht, R.; Hendry, E.; Shan, J.; Heinz, T. F.; Bonn, M. Carrier Dynamics in Semiconductors Studied with Time-Resolved Terahertz Spectroscopy. Rev. Mod. Phys. 2011, 83 (2), 543-586.

(30) Jnawali, G.; Rao, Y.; Yan, H.; Heinz, T. F. Observation of a Transient Decrease in Terahertz Conductivity of Single-Layer Graphene Induced by Ultrafast Optical Excitation. Nano Lett. 2013, 13 (2), 524-530.

(31) Frenzel, A. J.; Lui, C. H.; Fang, W.; Nair, N. L.; Herring, P. K.; Jarillo-Herrero, P.; Kong, J.; Gedik, N. Observation of Suppressed Terahertz Absorption in Photoexcited Graphene. Appl. Phys. Lett. 2013, 102 (11), 113111.

(32) Tielrooij, K. J.; Song, J. C. W.; Jensen, S. A.; Centeno, A.; Pesquera, A.; Zurutuza Elorza, A.; Bonn, M.; Levitov, L. S.; Koppens, F. H. L. Photoexcitation Cascade and Multiple Hot-Carrier Generation in Graphene. Nat. Phys. 2013, 9 (4), 248-252.

(33) Wang, H. I.; Braatz, M.-L.; Richter, N.; Tielrooij, K.-J.; Mics, Z.; Lu, H.; Weber, N.-E.; Müllen, K.; Turchinovich, D.; Kläui, M.; et al. Reversible Photochemical Control of Doping Levels in Supported Graphene. J. Phys. Chem. C 2017, 121 (7), 4083-4091.
(34) Hafez, H. A.; Chai, X.; Sekine, Y.; Takamura, M.; Oguri, K.; AlNaib, I.; Dignam, M. M.; Hibino, H.; Ozaki, T. Effects of Environmental Conditions on the Ultrafast Carrier Dynamics in Graphene Revealed by Terahertz Spectroscopy. Phys. Rev. B: Condens. Matter Mater. Phys. 2017, 95 (16), 165428.

(35) Conway, B. E. Ionic Hydration in Chemistry and Biophysics; Elsevier: Amsterdam, 1981.

(36) Feng, J.; Liu, K.; Graf, M.; Dumcenco, D.; Kis, A.; Di Ventra, M.; Radenovic, A. Observation of Ionic Coulomb Blockade in Nanopores. Nat. Mater. 2016, 15 (8), 850-855.

(37) Rollings, R. C.; Kuan, A. T.; Golovchenko, J. A. Ion Selectivity of Graphene Nanopores. Nat. Commun. 2016, 7, 11408.

(38) Chen, L.; Shi, G.; Shen, J.; Peng, B.; Zhang, B.; Wang, Y.; Bian, F.; Wang, J.; Li, D.; Qian, Z.; et al. Ion Sieving in Graphene Oxide Membranes via Cationic Control of Interlayer Spacing. Nature 2017, 550 (7676), 380-383.

(39) Friesen, S.; Hefter, G.; Buchner, R. Cation Hydration and Ion Pairing in Aqueous Solutions of $\mathrm{MgCl}_{2}$ and $\mathrm{CaCl}_{2}$. J. Phys. Chem. $B$ 2019, 123 (4), 891-900.

(40) Kresse, G.; Furthmüller, J. Efficiency of ab-Initio Total Energy Calculations for Metals and Semiconductors Using a Plane-Wave Basis Set. Comput. Mater. Sci. 1996, 6 (1), 15-50.

(41) Kresse, G.; Furthmüller, J. Efficient Iterative Schemes for Ab Initio Total-Energy Calculations Using a Plane-Wave Basis Set. Phys. Rev. B: Condens. Matter Mater. Phys. 1996, 54 (16), 11169-11186.

(42) Perdew, J. P.; Burke, K.; Ernzerhof, M. Generalized Gradient Approximation Made Simple. Phys. Rev. Lett. 1996, 77 (18), 38653868.

(43) Blöchl, P. E. Projector Augmented-Wave Method. Phys. Rev. B: Condens. Matter Mater. Phys. 1994, 50 (24), 17953-17979.

(44) Wright, A. F.; Leadbetter, A. J. The Structures of the $\beta$ Cristobalite Phases of $\mathrm{SiO}_{2}$ and $\mathrm{AlPO}_{4}$. Philos. Mag. 1975, 31 (6), 1391-1401.

(45) Yang, J.; Meng, S.; Xu, L.; Wang, E. G. Water Adsorption on Hydroxylated Silica Surfaces Studied Using the Density Functional Theory. Phys. Rev. B: Condens. Matter Mater. Phys. 2005, 71 (3), 035413. 